\title{
A narrative review of the roles of indoleamine 2,3-dioxygenase and tryptophan-2,3-dioxygenase in liver diseases
}

\author{
Qihui Zhou^, Yu Shi^, Chao Chen^, Fengtian Wu^, Zhi Chen^ \\ Department of Infectious Diseases, State Key Laboratory for Diagnosis and Treatment of Infectious Diseases, National Clinical Research Center for \\ Infectious Diseases, Collaborative Innovation Center for Diagnosis and Treatment of Infectious Diseases, the First Affiliated Hospital, College of \\ Medicine, Zhejiang University, Hangzhou, China \\ Contributions: (I) Conception and design: Q Zhou; (II) Administrative support: Z Chen; (III) Provision of study materials or patients: None; (IV) \\ Collection and assembly of data: Y Shi, C Chen, F Wu; (V) Data analysis and interpretation: None; (VI) Manuscript writing: All authors; (VII) Final \\ approval of manuscript: All authors. \\ Correspondence to: Zhi Chen, MD, PhD. Professor, Department of Infectious Diseases, State Key Laboratory for Diagnosis and Treatment \\ of Infectious Diseases, National Clinical Research Center for Infectious Diseases, Collaborative Innovation Center for Diagnosis and \\ Treatment of Infectious Diseases, the First Affiliated Hospital, College of Medicine, Zhejiang University, Hangzhou 310000 , China. \\ Email: zjuchenzhi@zju.edu.cn.
}

\begin{abstract}
Indoleamine 2,3-dioxygenase (IDO) and tryptophan-2,3-dioxygenase (TDO) are induced by several immune factors, such as interferon- $\gamma$, and act as intracellular enzymes that catabolize essential amino acid tryptophan into kynurenine and other downstream metabolites, including kynurenic acid (KYNA), xanthurenic acid (XA) and so on. IDO and TDO work as a double-edge sword. On one hand, they exert the immunomodulatory effects, especially immunosuppressive effects on the microenvironment including infections, pregnancy, tumor cells escape and transplantation. TDO plays the major role under basal conditions, while IDO comes into play under different circumstances of immune activation, thus IDO has a wider spectrum of immune regulation. On the other hand, these enzymes also inhibit pathogens such as Chlamydia pneumoniae, Staphylococcus aureus, Toxoplasma gondii and so on. Moreover, IDO regulates metabolic health through shaping intestinal microbiota. Recently, these enzymes have attracted more and more attention in liver diseases. Several studies have indicated that IDO and TDO can modulate viral hepatitis, autoimmune liver diseases, non-alcoholic fatty liver disease (NAFLD), liver cirrhosis, liver cancer even liver transplantation. Targeting them or their antagonists may provide novel therapeutic treatments for liver diseases. In this review, we will discuss the exact roles that IDO and TDO play in diverse hepatic diseases.
\end{abstract}

Keywords: Indoleamine 2,3-dioxygenase (IDO); viral hepatitis; autoimmune liver diseases; liver fibrosis and cirrhosis; liver tumors

Submitted Apr 28, 2020. Accepted for publication Oct 26, 2020.

doi: $10.21037 /$ atm-20-3594

View this article at: http://dx.doi.org/10.21037/atm-20-3594

\footnotetext{
^ ORCID: Qihui Zhou, 0000-0001-5369-3320; Yu Shi, 0000-0002-9392-7176; Chao Chen, 0000-0002-5346-3389; Fengtian Wu, 00000001-5047-3701; Zhi Chen, 0000-0002-0848-1502.
} 


\section{Introduction}

Kynurenine pathway (KP) accounts for $95 \%$ of the metabolic process of degradation of the essential amino acid L-tryptophan (Trp), which mainly occurs in the liver (1). The process is rate limited by tryptophan-2,3dioxygenase (TDO, also known as tryptophan pyrrolase) in the liver and indoleamine 2,3-dioxygenase (IDO) outside of the liver, especially in mucosal tissues and the immune system (2), including lung, placenta and immune cells (1). $\mathrm{L}$-Trp is first converted into $\mathrm{N}$-formylkynurenine, which is mediated by IDO or TDO, and then into L-kynurenine (3). L-Kynurenine is mainly metabolized into 3-hydroxykynurenine (3-HK) by kynurenine-3monooxygenase (KMO) and then further converted into 3-hydroxyanthranilic acid (3-HAA) by kynureninase (KYNU). 3-HAA is catabolized into quinolinic acid (QuinA) by 3-hydroxyanthranilate 3,4-dioxygenase (HAAO) and then processed into end product nicotinamide adenine dinucleotide (NAD+) by quinolinic acid phosphoribosyltransferase (QPRT). The catabolism of 3-HK into xanthurenic acid (XA) by kynurenine aminotransferases (KATs) and the metabolism of L-kynurenine into anthranilic acid (AA) and kynurenic acid (KYNA) are the minor L-Kynurenine metabolism pathways $(4,5)$ (Figure 1). TDO responds to Trp in meals and hormones such as glucocorticoids (5). Two forms of IDO, IDO1 and IDO2 are constitutively and locally expressed, respectively (1). The capacity of IDO2 to metabolize Trp is much smaller than that of IDO1, so we mainly focus on IDO1, and use IDO instead (IDO in the following article all refers to IDO1). IDO can be induced by interferon- $\gamma$ (IFN- $\gamma$ ), tumor necrotic factor (TNF- $\alpha$ ), lipopolysaccharide (LPS), and other proinflammatory cytokines in epithelial cells, dendritic cells (DCs) and macrophages (1,6-8). The combination of cytotoxic T-lymphocyte-associated protein-4 (CTLA-4) and CD80/CD86 molecules can also promote IDO expression (3). Moreover, anti-inflammatory cytokines hamper IDO expression $(1,9,10)$. The capacity of TDO in the liver to catalyze Trp is much stronger than that of IDO under basal conditions. However, IDO expressed in the extrahepatic tissues is markedly induced by the abovementioned cytokines under some pathophysiological circumstances of immune activation including bacterial and viral infections in the liver, as well as nonpathogenic inflammation including autoimmune liver diseases, liver tumors and liver transplantation, which means IDO has a wider range of effects. That is the reason why IDO could play a role in the liver diseases $(1,5)$. IDO has received more attention for its anti-infection and tumorpromoting activities (11).

The functions of the KP are usually as follows: (I) protection from excess Trp, which is exclusive to liverexpressed TDO; (II) c onservation of plasma Trp validity by TDO under normal conditions and by IDO under immune activation status; (III) production of kynurenine metabolites that take part in neuronal and immune responses (1). IDO is a double-edged sword. It plays the role of immunosuppressor in circumstances including infections, pregnancy, tumor cells escape and transplantation $(3,12)$. Indeed, it is both the depletion of Trp and the production of kynurenine and other metabolites that play the roles in immune regulation. Firstly, catabolizing Trp itself in the local microenvironment triggers amino acid-sensing signal transduction pathways. Secondly, the downstream metabolites of Trp reduce activities of innate cells like natural killer (NK) cells, DCs and macrophages, inhibit Th1 cell proliferation while promoting Th2-phenotype polarization, and induce transforming growth factor- $\beta$ (TGF- $\beta$ ) production and subsequent regulatory $\mathrm{T}$ cell (Treg) differentiation. Tregs activate myeloid-derived suppressor cells (MDSCs), leading to T cell inhibition. The metabolites also act to block the differentiation of type 17 T helper (Th17) cells (5,12-19) (Figure 2). IDO also inhibits intracellular pathogens like Cblamydia pneumoniae and bacteria like group B streptococci and mycobacteria $(6,8)$. TDO expressed on HeLa cells is able to suppress Staphylococcus aureus, Toxoplasma gondii and herpes simplex virus (18). However, IDO may have opposite antimicrobial effect when excessive Trp is available. Lepiller et al. (19) showed IDO promoted HIV persistence. Moreover, genetic deficiency of IDO could promote the metabolic health through shaping the intestinal microbiota. Depletion of microbiota in the high fat diet (HFD)-fed wildtype (WT) and IDO-knockout (KO) mice discarded the body weight difference between these two groups of mice in which IDO-KO mice were protected from metabolic complications. Co-housing these two groups of mice made the phenotype of WT mice similar to IDO-KO mice in the separated cages. What's more, feeding the WT mice with the feces from L-1methyl tryptophan (1MT)-treated ob/ob mice made the WT mice gain less body weight. The bacterial components of feces indicated that the decrease of Clostridiales Lachnospiraceae may be beneficial to control inflammation in the HFD-fed IDO-KO and 1MTtreated ob/ob mice. All these phenomena demonstrated that the intestinal microbiota of IDO-KO mice indeed 

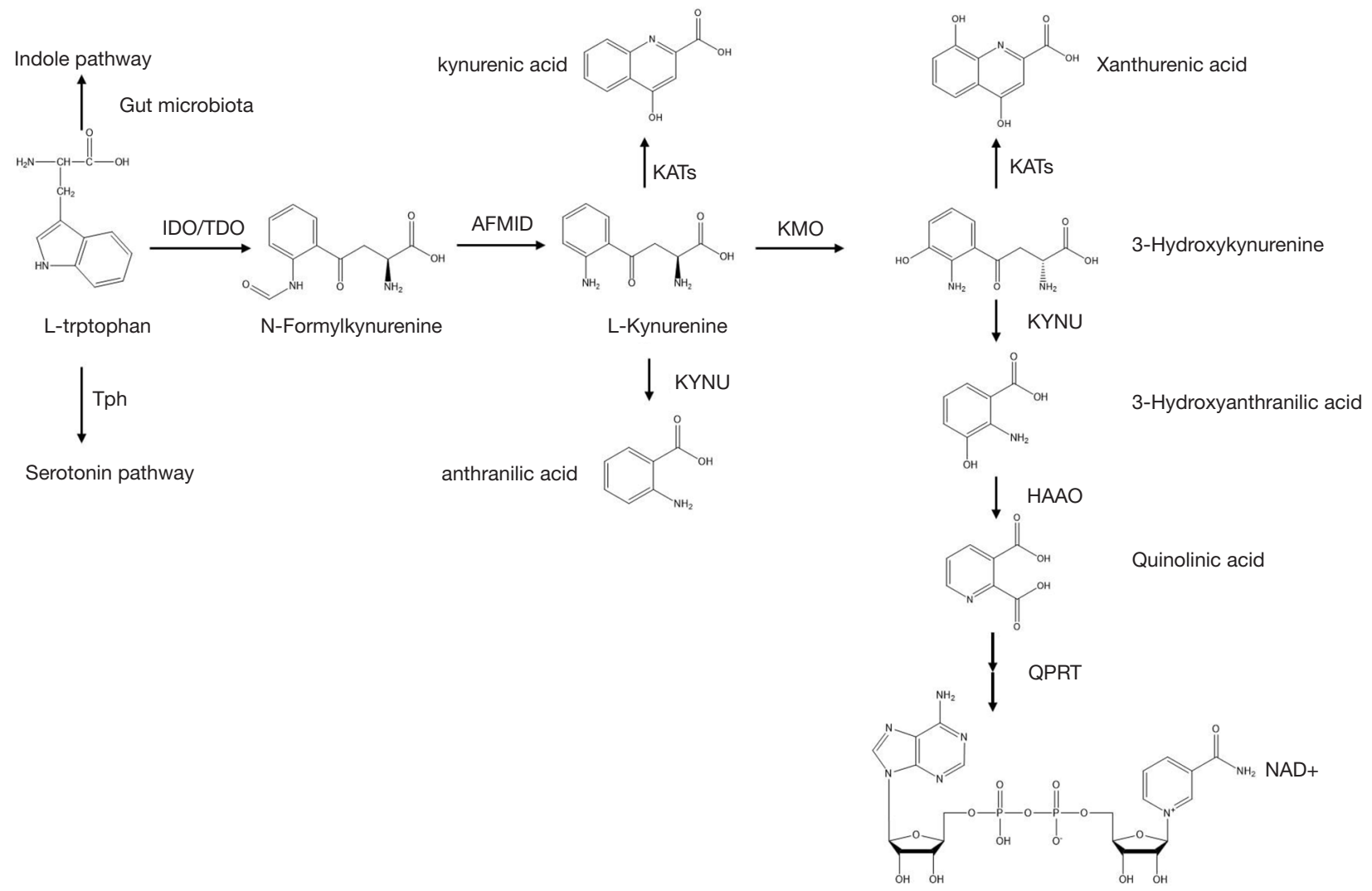

Figure 1 Degradation of tryptophan in kynurenine pathway. L-Trp is first converted into N-formylkynurenine, which is mediated by IDO or TDO, and then into L-kynurenine (3). L-Kynurenine is mainly metabolized into 3-hydroxykynurenine by KMO and then further converted into 3-hydroxyanthranilic acid by KYNU. 3-hydroxyanthranilic acid is catabolized into quinolinic acid by HAAO and then processed into end product NAD+ by QPRT. The catabolism of 3-hydroxykynurenine into xanthurenic acid by KATs and the metabolism of L-kynurenine into anthranilic acid and kynurenic acid are the minor L-kynurenine metabolism pathways. IDO, indoleamine 2,3-dioxygenase; TDO, tryptophan 2,3-dioxygenase; AFMID, kynurenine formamidase (arylformamidase); KMO, kynurenine-3-monooxygenase; KYNU, kynureninase; HAAO, 3-hydroxyanthranilate 3,4-dioxygenase; NAD+, nicotinamide adenine dinucleotide; QPRT, quinolinic aacid phosphoribosyltransferase; KATs, kynurenine aminotransferases.

protected the mice against obesity (20). In addition to the abovementioned effects, crucial roles for these enzymes in hepatic inflammation, fibrosis and cancers have also been suggested by a few studies. Herein, we provide an overview of the IDO/TDO in various hepatic diseases (Table 1).

We present the following article in accordance with the Narrative Review reporting checklist (available at: http:// dx.doi.org/10.21037/atm-20-3594).

\section{IDO/TDO in liver diseases}

\section{IDO/TDO in viral bepatitis}

Hepatitis B virus (HBV) and hepatitis $\mathrm{C}$ virus (HCV) infections are often regarded as the most common causes of viral hepatitis. Activated $\mathrm{HBV}$ and $\mathrm{HCV}$-specific cytotoxic T lymphocytes (CTLs) produced IFN- $\gamma$ which acted on hepatocytes, making the hepatocytes express IDO in patients with the corresponding type of viral hepatitis $(21,22)$. IDO has dual roles in antiviral responses; although IDO inhibited HBV replication, Trp consumption mediated by IDO suppressed the killing infected hepatocytes by CTLs (23). An increased level of IDO, together with chemokine (C-X-C motif) ligand (CXCL) 9, CXCL10 and CXCL11 expression, was the marker of HBV clearance in patients with acute hepatitis B (AHB) (6). Whereas, the anti-HBV role of IDO was lost in IDO-knockout (KO) cells (6). Moreover, the anti-HBV activity was enhanced 


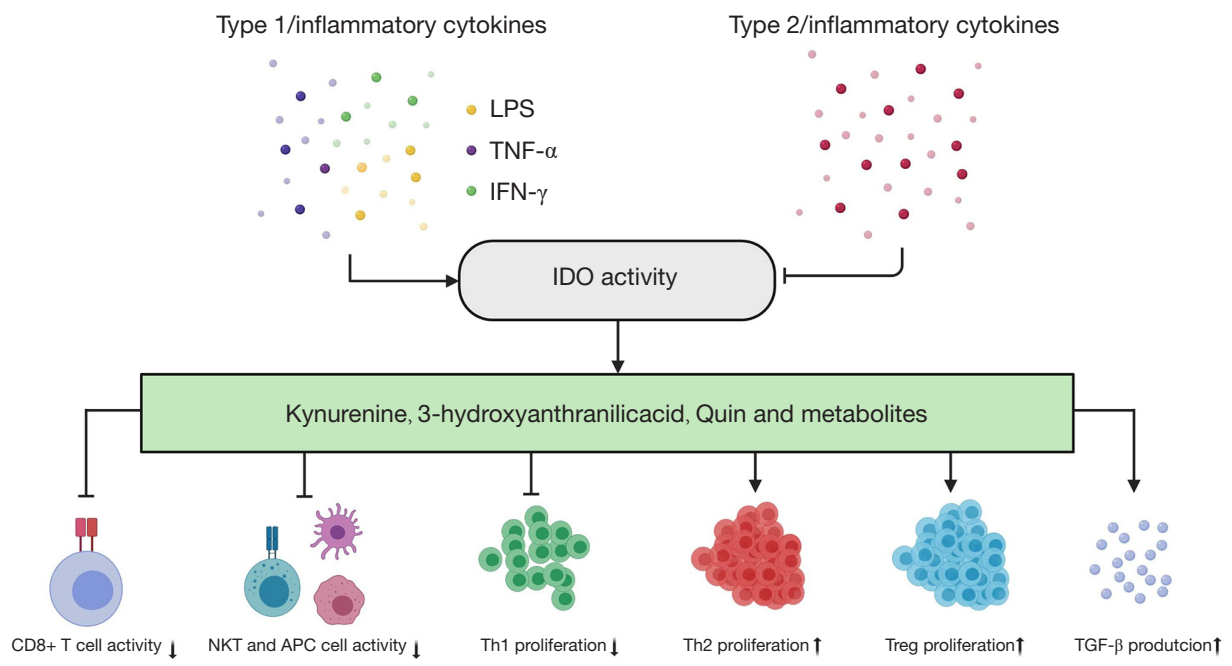

Figure 2 Modulation of kynurenine pathway in extrahepatic immune system. The activity of IDO can be stimulated by inflammatory cytokines and blocked by anti-inflammatory cytokines. The metabolites of KP catabolized by IDO play the suppressive role in the immune responses. They reduce activities of CD8+T cells and innate cells such as NKT cells, DCs and macrophages. They also inhibit Th1 cell proliferation. However, They promote Th2-phenotype polarization and Treg differentiation, as well as TGF- $\beta$ production.

Table 1 Role of IDO/TDO in liver diseases

\begin{tabular}{ll}
\hline Liver diseases & Roles of IDO/TDO \\
\hline Viral hepatitis & Inhibition of HBV replication; Exacerbation of liver injury \\
Autoimmune liver diseases & Protection against immune overactivation \\
Non-alcoholic fatty liver disease & Protection against liver inflammation; Promotion of liver steatosis \\
Liver fibrosis and cirrhosis & Aggravation or attenuation of liver lesions \\
Liver tumors & Promotion of tumor progression \\
Hepatectomy and liver transplantation & Suppression of liver regeneration; Protection against immune rejection \\
\hline
\end{tabular}

by NK cells and plasmacytoid dendritic cells (pDC) by driving the production of IFN- $\gamma$ in $\mathrm{HBV}$-positive Huh7 cells (6). Mao et al. (23) also demonstrated that IDO, one of 37 IFN-stimulated genes (ISGs), was able to dosedependently inhibit intracellular HBV DNA replication in HepG2 cell lines but not RNA transcription. However, IDO was also a double-edged sword. Among chronic HBV infection, especially $\mathrm{HBeAg}(+)$ patients, persistent infection and immune tolerance might be mediated by MDSCs through expression of IDO, thus leading to HBsAg-specific T cells suppression (24). IDO exacerbated liver injury in the mice model of acute hepatitis injected with HBVspecific CTLs, and liver injury was eased when the mice were treated with its antagonist 1-methyl-D-tryptophan (1MT) (25). In fulminant viral hepatitis, the activity of IDO and metabolites of $\mathrm{KP}$ were positively correlated with liver injury (25).

In regard to patients with $\mathrm{HCV}$ infection, the expression of IFN- $\gamma$ and IDO was induced (26). It was reported that the hepatic mRNA expression of IDO was upregulated when chimpanzees were acutely infected and remained chronically high (19). Moreover, the enhanced IDO activity was positively correlated with severity of hepatic inflammation and fibrosis (27). Similar to the functions of IDO in HBV infection, IDO also played an important role in anti-HCV and immunoregulation in different phases of $\mathrm{HCV}$ infection. For instance, upregulated expression of IDO in the early stage inhibited HCV replication, while IDO might induce immune tolerance in the later time (19). One of mechanisms underlying the immune tolerance 
can be attributed to the functions of IDO expressed by DCs mediated through the suppression of activated $T$ cell and induction of Tregs (27). The key question of how the antiviral effect is balanced with its immunosuppressive role can be answered by evaluating the levels of Trp (19).

When it comes to other hepatitis virus infections, blockage of TDO and IDO lead to different outcomes. In a study of mouse hepatitis virus (MHV-A59) model, blocking TDO with LM10, a derivative of 3-[2-(pyridyl) ethenyl] indole, decreased the level of anti-MHV antibody and inhibited autoimmune responses, indicating that TDO may be a new perspective and target to treat certain kind of viral infection (28). Nevertheless, Duhalde Vega et al. (29) expressed the opposite opinion, indicating that application of L-MT, which blocked IDO without affecting TDO, did not protect the mice from MHV infection but instead augmented detrimental effects of the virus action, including hypergammaglobulinemia, anti-MHV $\mathrm{Ab}$ and uric acid release, as well as liver fibrosis.

\section{IDO in autoimmune liver diseases}

Autoimmune liver diseases are chronic liver diseases caused by immune dysfunction. In a model of $\alpha$-galactosylceramide $(\alpha$-GalCer) - induced hepatitis that resembles pathogenesis of autoimmune liver disease, IDO acted as liver protector against immune overactivation (3). Among patients with primary biliary cirrhosis ( $\mathrm{PBC})$, there was high degree of kynurenine metabolized from tryptophan and it increased the proportion of Tregs (30). Although few studies have been published, the role of IDO in autoimmune liver diseases cannot be ignored.

\section{IDO in non-alcobolic fatty liver disease (NAFLD)}

NAFLD is one of the metabolic diseases with high prevalence among contemporary humans. It was reported that IDO-KO mice were more prone to liver inflammation and fibrosis induced by a HFD compared to IDO-WT, suggesting that IDO may be beneficial in NAFLD (31). Moreover, a study conducted among obese women showed that the increased activity of IDO in the liver and adipose tissue was inversely correlated with balance of Th17 relative to Treg in subcutaneous fat (32). However, Laurans et al. (20) demonstrated that upregulated activity of IDO was correlated with obesity. They found that lack of IDO ameliorated the state of insulin resistance, improved the intestinal permeability, and protected mice from liver steatosis. In addition, another study demonstrated that kynurenine, the metabolite of Trp by IDO, was a known aryl hydrocarbon receptor (AhR) agonist and resulted in obesity. Blocking IDO or AhR prevented longer-term, diet-induced liver steatosis (33). Moreover, a HFD which facilitated proinflammatory environment suppressed the activity of TDO in the liver, but enhanced the extrahepatic metabolism of Trp by IDO (34). Therefore, IDO plays different roles in the NAFLD which needs further research.

\section{IDO in liver fibrosis and cirrbosis}

Liver fibrosis, which may progress to liver cirrhosis as the disease continues to advance, is characterized by extracellular matrix (ECM) component accumulation. IDO is found to be associated with liver fibrosis and cirrhosis. Zhong et al. (9) demonstrated that the serum level of IDO was positively related to liver lesions, regardless of hepatic function or liver stiffness, in liver cirrhosis patients and mice. Asghar et al. (35) found that it was more highly expressed in HCV-infected patients than in healthy subjects, which contributed to HCVrelated liver cirrhosis. Another study showed that IFN- $\gamma$ along with IDO inhibitor 1-MT induced hepatic stellate cells (HSCs) apoptosis, leading to amelioration of liver fibrosis (36). In addition, IDO-KO mice were protected from liver fibrosis with a reduced level of IL-17A and an increased level of TDO. These findings suggested that IDO might be a sign of liver cirrhosis. However, Ogiso et al. (37) came to the opposite conclusion, reporting that liver inflammation and fibrosis were intensified in IDO-KO mice treated with carbon tetrachloride $\left(\mathrm{CCL}_{4}\right)$ for 6 weeks. In a study which focused on utilizing mesenchymal stem cells (MSC) to mitigate liver fibrosis, Milosavljevic et al. (38) demonstrated that IDO was required to restrain IL-17 production by Th17 cells, thus attenuating liver fibrosis. Taken together, the opposite effects of IDO on liver fibrosis were associated with different circumstances. It exacerbated liver fibrosis and cirrhosis in some cases, and in other cases, it ameliorated the lesions.

\section{IDO/TDO in liver tumors}

Liver tumors are one of the most common cancers worldwide. Many chronic liver diseases, such as viral infections and alcoholic liver diseases, lead to pathogenesis of liver cancers. IDO acts as immunosuppressors in malignancies (39). A previous study found that the IDO was positively correlated with tumor progression. It has 
been demonstrated that hepatocellular carcinoma (HCC) cells and surrounding cells expressed high levels of IDO, which helped tumors escape immune surveillance (40). Monocytes/macrophages also produced proinflammatory cytokines to induce IDO expression upon encountering CD69+T cells, leading to tumor growth $(41,42)$. In addition, the frequency of these monocytes/macrophages in the tumor site was negatively correlated with survival in HCC (41). Apart from monocytes/macrophages, a clinical study also demonstrated an elevated percentage of intratumoral neutrophils infiltration and increased expression of IDO in HCC cells. IDO and intratumoral neutrophils may predict the prognosis of overall survival in HCC (43). IDO participated in the process of tumor antigen tolerance by contributing to the presentation of tumor antigens by host antigen-presenting cells (APCs), the escape from surveillance mediated by immune cells and induction of $\mathrm{T}$ cells exhaustion in the tumor microenvironment $(8,44)$. Hypoxia inducible factor-1 $\alpha$ (HIF-1 $\alpha$ )-CCL20-IDO pathway promoted tumor metastasis as well (44). Furthermore, in a model of diethylnitrosamine-induced liver carcinogenesis, IDO$\mathrm{KO}$ mice developed fewer hepatic tumor foci and fewer Tregs infiltrated in the liver compared to IDO-WT mice. This finding indicated that inhibiting IDO or reducing the levels of downstream metabolites of KP may be beneficial to HCC treatment (45). Brown et al. (46) demonstrated that application of immune checkpoint (IC) inhibitors increased IDO expression in HCC patients, which made the drugs less effective than expected. Brown et al. (46) and $\mathrm{Li}$ et al. (47) suggested that a therapeutic regimen consisting of IC inhibitors and IDO inhibitors may be beneficial in improving treatment efficacy. Not only IDO, but also TDO played the immunosuppressive role in the malignancy (48). Canavese et al. (49) supp orted the hypothesis that TDO-Kyn-AhR metabolic pathway led to immunosuppressive effect, thus promoting HCC development. Tumor cells proliferated and escaped immune surveillance in the presence of a high level of kynurenine via two mechanisms (5). First, AhR activated by KP metabolites promoted cell migration by TDO; second, AhR disrupted $\mathrm{T}$ cell proliferation (50). TDO inhibition may change an unresponsive tumor immune microenvironment and enhance antitumor ability with minimal harmful effects on a normal cell line, which offered a potential way to treat HCC $(48,51)$. Therefore, IDO helps liver tumor escape the immune surveillance and promotes tumor progression.

\section{IDO in bepatectomy and liver transplantation}

IDO also played important roles in hepatectomy and liver transplantation. After partial hepatectomy, IDO deficiency promoted activation of immune cells and broke immunotolerance, thus leading to liver regeneration (52). In regards to liver transplantation, IDO expression is upregulated in the APCs in rat allograft liver $(53,54)$. For instance, the expression of IDO by Kupffer cells (KCs) in tolerance group grew dependently over time (55). Moreover, Sun et al. (56) demonstrated that IFN- $\gamma$-treated IDO-expressing DCs re-infused rat group showed milder symptoms of acute rejection compared to the control group. These findings indicated that IDO can be used to protect against immune rejection in liver transplantation. In addition, transfusion of IDO accompanied with cytotoxic lymphocyte antigen 4-immunoglobulin (CTLA4-Ig) gene increased survival rate in a rat model of liver transplantation acute rejection (57). Taken together, IDO induces immune tolerance and plays a protective role in liver transplantation.

\section{Conclusions}

Overall, the metabolic enzymes IDO or TDO in the KP of trptophan degradation have recently been recognized as important mediators that function as double-edge swords in various liver diseases. We may be able to take advantage of the predicted and therapeutic roles of these enzymes and their antagonists to prevent and treat hepatic diseases in the near future.

\section{Acknowledgments}

Authors are grateful to the Department of Infectious Diseases, State Key Laboratory for Diagnosis and Treatment of Infectious Diseases, National Clinical Research Center for Infectious Diseases, Collaborative Innovation Center for Diagnosis and Treatment of Infectious Diseases, the First Affiliated Hospital, College of Medicine, Zhejiang University for their contribution to this manuscript.

Funding: This work was supported by the National Science and Technology Major Project of China [grant number 2018ZX10302206, 2017ZX10202203].

\section{Footnote}

Reporting Checklist: The authors have completed the 
Narrative Review reporting checklist. Available at http:// dx.doi.org/10.21037/atm-20-3594

Peer Review File: Available at http://dx.doi.org/10.21037/ atm-20-3594

Conflicts of Interest: All authors have completed the ICMJE uniform disclosure form (available at http://dx.doi. org/10.21037/atm-20-3594). The authors have no conflicts of interest to declare.

Ethical Statement: The authors are accountable for all aspects of the work in ensuring that questions related to the accuracy or integrity of any part of the work are appropriately investigated and resolved.

Open Access Statement: This is an Open Access article distributed in accordance with the Creative Commons Attribution-NonCommercial-NoDerivs 4.0 International License (CC BY-NC-ND 4.0), which permits the noncommercial replication and distribution of the article with the strict proviso that no changes or edits are made and the original work is properly cited (including links to both the formal publication through the relevant DOI and the license). See: https://creativecommons.org/licenses/by-nc-nd/4.0/.

\section{References}

1. Badawy AA. Kynurenine Pathway of Tryptophan Metabolism: Regulatory and Functional Aspects. Int J Tryptophan Res 2017;10:1178646917691938.

2. Pallotta MT, Orabona C, Volpi C, et al. Indoleamine 2,3-dioxygenase is a signaling protein in long-term tolerance by dendritic cells. Nat Immunol 2011;12:870-8.

3. Ito $\mathrm{H}$, Hoshi $\mathrm{M}$, Ohtaki $\mathrm{H}$, et al. Ability of IDO to attenuate liver injury in alpha-galactosylceramide-induced hepatitis model. J Immunol 2010;185:4554-60.

4. Walczak K, Wnorowski A, Turski WA, et al. Kynurenic acid and cancer: facts and controversies. Cell Mol Life Sci 2020;77:1531-50.

5. Cervenka I, Agudelo LZ, Ruas JL. Kynurenines: Tryptophan's metabolites in exercise, inflammation, and mental health. Science 2017;357:eaaf9794.

6. Yoshio S, Sugiyama M, Shoji H, et al. Indoleamine-2,3dioxygenase as an effector and an indicator of protective immune responses in patients with acute hepatitis B. Hepatology 2016;63:83-94.

7. Wirthgen E, Tuchscherer M, Otten W, et al. Activation of indoleamine 2,3-dioxygenase by LPS in a porcine model. Innate Immun 2014;20:30-9.

8. Mellor AL, Munn DH. IDO expression by dendritic cells: tolerance and tryptophan catabolism. Nat Rev Immunol 2004;4:762-74.

9. Zhong $\mathrm{W}$, Gao L, Zhou Z, et al. Indoleamine 2,3-dioxygenase 1 deficiency attenuates CCl4-induced fibrosis through Th17 cells down-regulation and tryptophan 2,3-dioxygenase compensation. Oncotarget 2017;8:40486-500.

10. Hassanain HH, Chon SY, Gupta SL. Differential regulation of human indoleamine 2,3-dioxygenase gene expression by interferons-gamma and -alpha. Analysis of the regulatory region of the gene and identification of an interferon-gamma-inducible DNA-binding factor. J Biol Chem 1993;268:5077-84.

11. Valladares R, Bojilova L, Potts AH, et al. Lactobacillus johnsonii inhibits indoleamine 2,3-dioxygenase and alters tryptophan metabolite levels in BioBreeding rats. Faseb J 2013;27:1711-20.

12. Wang $M$, Dong $X$, Huang $Y$, et al. Activation of the kynurenine pathway is associated with poor outcome in Pneumocystis pneumonia patients infected with HIV: results of 2 months cohort study. BMC Infect Dis 2019;19:223.

13. Frumento G, Rotondo R, Tonetti M, et al. Tryptophanderived catabolites are responsible for inhibition of $\mathrm{T}$ and natural killer cell proliferation induced by indoleamine 2,3-dioxygenase. J Exp Med 2002;196:459-68.

14. Andersen MH. Anti-regulatory T cells. Semin Immunopathol 2017;39:317-26.

15. Hill M, Tanguy-Royer S, Royer P, et al. IDO expands human $\mathrm{CD} 4+\mathrm{CD} 25$ high regulatory $\mathrm{T}$ cells by promoting maturation of LPS-treated dendritic cells. Eur J Immunol 2007;37:3054-62.

16. Savitz J. The kynurenine pathway: a finger in every pie. Mol Psychiatry 2020;25:131-47.

17. Kesarwani P, Kant S, Prabhu A, et al. The interplay between metabolic remodeling and immune regulation in glioblastoma. Neuro Oncol 2017;19:1308-15.

18. Mondanelli G, Iacono A, Carvalho A, et al. Amino acid metabolism as drug target in autoimmune diseases. Autoimmun Rev 2019;18:334-48.

19. Lepiller Q, Soulier E, Li Q, et al. Antiviral and Immunoregulatory Effects of Indoleamine-2,3Dioxygenase in Hepatitis C Virus Infection. J Innate Immun 2015;7:530-44.

20. Laurans L, Venteclef N, Haddad Y, et al. Genetic 
deficiency of indoleamine 2,3-dioxygenase promotes gut microbiota-mediated metabolic health. Nat Med 2018;24:1113-20.

21. Iwamoto N, Ito $H$, Ando K, et al. Upregulation of indoleamine 2,3-dioxygenase in hepatocyte during acute hepatitis caused by hepatitis B virus-specific cytotoxic T lymphocytes in vivo. Liver Int 2009;29:277-83.

22. Larrea E, Riezu-BojJI, Gil-Guerrero L, et al. Upregulation of indoleamine 2,3-dioxygenase in hepatitis C virus infection. J Virol 2007;81:3662-6.

23. Mao R, Zhang J, Jiang D, et al. Indoleamine 2,3-dioxygenase mediates the antiviral effect of gamma interferon against hepatitis B virus in human hepatocytederived cells. J Virol 2011;85:1048-57.

24. Yang F, Yu X, Zhou C, et al. Hepatitis B e antigen induces the expansion of monocytic myeloid-derived suppressor cells to dampen T-cell function in chronic hepatitis B virus infection. PLoS Pathog 2019;15:e1007690.

25. Ohtaki H, Ito H, Ando K, et al. Kynurenine production mediated by indoleamine 2,3-dioxygenase aggravates liver injury in $\mathrm{HBV}$-specific CTL-induced fulminant hepatitis. Biochim Biophys Acta 2014;1842:1464-71.

26. Mehraj V, Routy JP. Tryptophan Catabolism in Chronic Viral Infections: Handling Uninvited Guests. Int J Tryptophan Res 2015;8:41-8.

27. Higashitani K, Kanto T, Kuroda S, et al. Association of enhanced activity of indoleamine 2,3-dioxygenase in dendritic cells with the induction of regulatory $\mathrm{T}$ cells in chronic hepatitis $\mathrm{C}$ infection. J Gastroenterol 2013;48:660-70.

28. Duhalde Vega M, Aparicio JL, Mandour MF, et al. The autoimmune response elicited by mouse hepatitis virus (MHV-A59) infection is modulated by liver tryptophan2,3-dioxygenase (TDO). Immunol Lett 2020;217:25-30.

29. Duhalde Vega M, Aparicio JL, Retegui LA. Levo-1-methyl tryptophan aggravates the effects of mouse hepatitis virus (MHV-A59) infection. Int Immunopharmacol 2015;24:377-82.

30. Asghar K, Brain J, Palmer JM, et al. Potential role of indoleamine 2,3-dioxygenase in primary biliary cirrhosis. Oncol Lett 2017;14:5497-504.

31. Nagano J, Shimizu M, Hara T, et al. Effects of indoleamine 2,3-dioxygenase deficiency on high-fat dietinduced hepatic inflammation. PLoS One 2013;8:e73404.

32. Wolowczuk I, Hennart B, Leloire A, et al. Tryptophan metabolism activation by indoleamine 2,3-dioxygenase in adipose tissue of obese women: an attempt to maintain immune homeostasis and vascular tone. Am J Physiol
Regul Integr Comp Physiol 2012;303:R135-43.

33. Moyer BJ, Rojas IY, Kerley-Hamilton JS, et al. Inhibition of the aryl hydrocarbon receptor prevents Western dietinduced obesity. Model for AHR activation by kynurenine via oxidized-LDL, TLR2/4, TGFbeta, and IDO1. Toxicol Appl Pharmacol 2016;300:13-24.

34. Badawy AA. Tryptophan availability for kynurenine pathway metabolism across the life span: Control mechanisms and focus on aging, exercise, diet and nutritional supplements. Neuropharmacology 2017;112:248-63.

35. Asghar K, Ashiq MT, Zulfiqar B, et al. Indoleamine 2,3-dioxygenase expression and activity in patients with hepatitis $\mathrm{C}$ virus-induced liver cirrhosis. Exp Ther Med 2015;9:901-4.

36. Oh JE, Shim KY, Lee JI, et al. 1-Methyl-L-tryptophan promotes the apoptosis of hepatic stellate cells arrested by interferon-gamma by increasing the expression of IFN-gammaRbeta, IRF-1 and FAS. Int J Mol Med 2017;40:576-82.

37. Ogiso H, Ito H, Ando T, et al. The Deficiency of Indoleamine 2,3-Dioxygenase Aggravates the CCl4-Induced Liver Fibrosis in Mice. PLoS One 2016;11:e0162183.

38. Milosavljevic N, Gazdic M, Simovic Markovic B, et al. Mesenchymal stem cells attenuate liver fibrosis by suppressing Th17 cells - an experimental study. Transpl Int 2018;31:102-15.

39. Munn DH, Mellor AL. IDO and tolerance to tumors. Trends Mol Med 2004;10:15-8.

40. Pan K, Wang H, Chen MS, et al. Expression and prognosis role of indoleamine 2,3-dioxygenase in hepatocellular carcinoma. J Cancer Res Clin Oncol 2008;134:1247-53.

41. Zhao Q, Kuang DM, Wu Y, et al. Activated CD69+ $T$ cells foster immune privilege by regulating IDO expression in tumor-associated macrophages. J Immunol 2012;188:1117-24.

42. Zhao Q, Wang PP, Huang ZL, et al. Tumoral indoleamine 2, 3-dioxygenase 1 is regulated by monocytes and $\mathrm{T}$ lymphocytes collaboration in hepatocellular carcinoma. Oncotarget 2016;7:14781-90.

43. Wang Y, Yao R, Zhang L, et al. IDO and intra-tumoral neutrophils were independent prognostic factors for overall survival for hepatocellular carcinoma. J Clin Lab Anal 2019;33:e22872.

44. Asghar K, Farooq A, Zulfiqar B, et al. Indoleamine 2,3-dioxygenase: As a potential prognostic marker and immunotherapeutic target for hepatocellular carcinoma. 
World J Gastroenterol 2017;23:2286-93.

45. Shibata Y, Hara T, Nagano J, et al. The Role of Indoleamine 2,3-Dioxygenase in DiethylnitrosamineInduced Liver Carcinogenesis. PLoS One 2016;11:e0146279.

46. Brown ZJ, Yu SJ, Heinrich B, et al. Indoleamine 2,3-dioxygenase provides adaptive resistance to immune checkpoint inhibitors in hepatocellular carcinoma. Cancer Immunol Immunother 2018;67:1305-15.

47. Li S, Han X, Lyu N, et al. Mechanism and prognostic value of indoleamine 2,3-dioxygenase 1 expressed in hepatocellular carcinoma. Cancer Sci 2018;109:3726-36.

48. Opitz CA, Litzenburger UM, Sahm F, et al. An endogenous tumour-promoting ligand of the human aryl hydrocarbon receptor. Nature 2011;478:197-203.

49. Canavese M, Wijesundara D, Maddern GJ, et al. Hepatitis $\mathrm{C}$ virus drives the pathogenesis of hepatocellular carcinoma: from immune evasion to carcinogenesis. Clin Transl Immunology 2016;5:e101.

50. Munn DH, Sharma MD, Baban B, et al. GCN2 kinase in $T$ cells mediates proliferative arrest and anergy induction in response to indoleamine 2,3-dioxygenase. Immunity 2005;22:633-42.

51. Hua S, Wang X, Chen F, et al. Novel conjugates with dual

Cite this article as: Zhou Q, Shi Y, Chen C, Wu F, Chen Z. A narrative review of the roles of indoleamine 2,3-dioxygenase and tryptophan-2,3-dioxygenase in liver diseases. Ann Transl Med 2021;9(2):174. doi: 10.21037/atm-20-3594 suppression of glutathione S-transferases and tryptophan2,3-dioxygenase activities for improving hepatocellular carcinoma therapy. Bioorg Chem 2019;92:103191.

52. Ogiso H, Ito H, Kanbe A, et al. The Inhibition of Indoleamine 2,3-Dioxygenase Accelerates Early Liver Regeneration in Mice After Partial Hepatectomy. Dig Dis Sci 2017;62:2386-96.

53. Lin YC, Chen CL, Nakano T, et al. Immunological role of indoleamine 2,3-dioxygenase in rat liver allograft rejection and tolerance. J Gastroenterol Hepatol 2008;23:e243-50.

54. Lin YC, Goto S, Tateno C, et al. Induction of indoleamine 2,3-dioxygenase in livers following hepatectomy prolongs survival of allogeneic hepatocytes after transplantation. Transplant Proc 2008;40:2706-8.

55. Luan X, Liao W, Lai X, et al. Dynamic changes of indoleamine 2,3-dioxygenase of Kupffer cells in rat liver transplant rejection and tolerance. Transplant Proc 2012;44:1045-7.

56. Sun X, Gong ZJ, Wang ZW, et al. IDO-competent-DCs induced by IFN-gamma attenuate acute rejection in rat liver transplantation. J Clin Immunol 2012;32:837-47.

57. Wu Y, Yu Z, Gong J, et al. Effects of combined genes of CTLA4Ig and IDO in post-liver transplantation immune tolerance of rats. Ann Hepatol 2016;15:729-37. 